\title{
A new fractional-order chaotic system with plane equilibrium: Bifurcation analysis, Multi-stability and DSP implementation
}

\author{
Tianming Liu ${ }^{1}$, Xiaoyu Zhang ${ }^{2}$, Peng Li $^{1, *}$, and Huizhen Yan $^{1}$ \\ \{lipeng@dlpu.edu.cn\} \\ ${ }^{1}$ School of Information Science and Engineering, Dalian polytechnic University, Dalian, 116034, China \\ ${ }^{2}$ Agronomy College, ShenYang Agricultural University, Shenyang, 110161, China
}

\begin{abstract}
In this paper, based on the definition of conformable differential, a 4D chaotic system is discretized by Adomian decomposition method (ADM) and its numerical solution is obtained. The stability of the system at the equilibrium point is analyzed. From the coexisting phase diagram, bifurcation model and Lyapunov exponential spectrum, the multi-stability of fractional chaotic systems is analyzed. Spectral entropy complexity is used to study the complex performance of fractional order systems. Then, according to the measurement result of spectrum entropy complexity, three sets of parameters with higher complexity are selected to design a pseudo-random number generator (PRNG). The designed PRNG passed all randomness tests, indicating that the system has high practical application value. Meanwhile, the related design of the circuit implementation of the chaotic system was completed and verified on the DSP platform. Research shows that fractional-order chaotic systems have special dynamic behaviors and multiple stability phenomena that evolve with q. The pseudo-random sequence generator and digital circuit designed in this research provide guidance for the application of fractional-order chaotic systems.
\end{abstract}

Keywords: Dynamics, CADM algorithm, Coexisting attractors, PRNG.

\section{Introduction}

Chaos has received widespread attention from scholars since its discovery, and it has now become an important part of the nonlinear field. Lorentz accidentally noticed the existence of chaos in the atmosphere and constructed the Lorentz system[1-4]. For a long time, people 
have been committed to building systems with stronger chaotic characteristics. The theory of fractional calculus is used to model and explain the phenomena of nature, which can reveal the dynamic behavior of the actual system more objectively. The fractional order system has great advantages in describing the memory and heredity of various systems[5]. Therefore, some scholars try to introduce the definition of fractional differential into chaotic systems. The definition of fractional differential was introduced into some typical chaotic systems, not only the phenomenon of chaos exists, but the chaotic characteristics of the system become more complicated[6-8]. For example, fractional-order Lorentz system[9], fractional-order Liu system[10], etc. These systems are now widely used in various applications, such as [11, 12], encryption applications[13, 14], biomedical engineering[15], etc.

In recent years, a peculiar phenomenon of coexistence attractors has been discovered in chaotic systems[16]. The typical characteristic of a chaotic system is its high sensitivity to initial conditions. However, some systems have some special phenomena, which need to ensure that a certain parameter remains unchanged. Different initial conditions make the system have multiple numerical solutions[17]. The existing research results show that some low-dimensional nonlinear differential equations will not only present chaotic phenomena, but also produce multiple coexisting attractors[18-21]. Ma et al. studied the multi-stability phenomenon in the chaotic system of Hopfield neural network[22]. Yu et al. proposed a new memristive hyperchaotic system with multiple stability and designed a circuit and chaotic synchronization control in Ref[23]. Liu et al. analyzed the chaotic system with infinite attractors and implemented DSP in Ref[24]. Compared with the research on coexistence attractors of integer order systems, the research literature on coexistence attractors of fractional order systems is relatively scarce. Compared with general fractional-order chaotic systems, fractional-order chaotic systems with coexistence phenomena can show better performance in the application field[25-28]. Nowadays, the multiple stability of fractional-order chaotic systems attracts scholars to further study[29, 30].

The algorithms for solving fractional chaotic systems are mainly frequency domain method (FDM), predictive correction method (ABM) and Adomian decomposition method (ADM)[31]. These algorithms usually use the Riemann-Liouville definition and Caputo definition[32]. Khalil et al. proposed the definition of consistent fractional differential[33], which combined with the ADM algorithm (CADM) to solve the fractional differential equations can overcome some of the shortcomings of the existing algorithms[34]. Compared with ADM algorithm, CADM algorithm has the advantages of fast convergence speed, fast calculation speed and less resource consumption [35]. At present, there are few studies on the use of improved ADM algorithm (CADM) to solve fractional chaotic systems. So far, many 
studies have been conducted on the application of fractional chaotic systems, such as nuclear magnetic resonance[36], cellular neural networks and secure communication[37]. The application of chaos relies on digital signal processing technology and the design of hardware circuits. Digital signal processor (DSP) has been widely used in engineering due to its excellent performance and convenient processing[38-40]. Therefore, this paper considers the realization of the chaotic system solving process on the DSP platform. C language is used for programming to obtain an approximate chaotic attractor phase diagram on digital circuits.

The rest of this paper is organized as follows: the definition of consistent fractional-order and the numerical solution of fractional chaotic systems are introduced in Sect 2. The dynamics of the system and its multiple stability are studied In Sect 3. In Sect 4, a pseudo-random number generator was designed and tested for randomness. In Sect 5, Realization of Fractional Chaos System on DSP. Finally, the results of this paper are summarized.

\section{Definition of Fractional Calculus and solution of Chaotic System}

\subsection{Conformable differential definition}

Conformable differential definition can be better combined with ADM decomposition algorithm for solving fractional differential equations. Based on this definition, the differential equation can be written as

$$
\left\{\begin{array}{l}
T_{\mathrm{t}}^{q} x(t)=L x(t)+N x(t)+g \\
x^{(k)}\left(t_{0}^{+}\right)=b_{k}, k=0, \cdots, m-1 \\
m \in N, m-1<q \leq m
\end{array}\right.
$$

here $* T_{t}^{t}$ is the derivative operator. The state variables describe as $x(t)=\left[x_{1}(t), x_{2}(t), \ldots, x(t)\right]^{\mathrm{T}}$. The initial value of the differential equation is expressed as $b_{k} . L$ and $N$ respectively represent the linear and nonlinear terms of the differential equation. After applying $T_{t}^{q}$ to both sides of Eq. (1), it can be described as

$$
x(t)=I_{t_{0}}^{q} L x(t)+I_{t_{0}}^{q} N x(t)+I_{t_{0}}^{q} g+x\left(t_{0}\right)
$$

where $* I_{t_{0}}^{\varphi}$ is the integral operator, Here, some properties of the integral operator need to be listed as follows 


$$
\begin{gathered}
I_{t_{0}}^{q} C=\frac{\left(t-t_{0}\right)^{q}}{q} C \\
I_{t_{0}}^{q_{n}} \ldots I_{t_{0}}^{q_{2}} I_{t_{0}}^{q_{1}} C=C \frac{\left(t-t_{0}\right)^{q_{1}+q_{2}+\ldots+q_{n}}}{q_{1}\left(q_{2}+q_{2}\right) \ldots\left(q_{1}+\ldots+q_{n}\right)}
\end{gathered}
$$

The discrete formula of the nonlinear term is

$$
\left\{\begin{array}{l}
A_{j}^{i}=\frac{1}{i !}\left[\frac{d^{i}}{d \lambda^{i}} N\left(v_{j}^{i}(\lambda)\right)\right]_{\lambda=0} \\
v_{j}^{i}(\lambda)=\sum_{k=0}^{i}(\lambda)^{k} x_{j}^{k}
\end{array}\right.
$$

here, $i=0,1, \ldots, \infty$ and $j=1,2, \ldots, n$. According to Equation 5 , the nonlinear term can be rewritten as

$$
N x=\sum_{i=0}^{\infty} A^{i}\left(x^{0}, x^{1}, \cdots, x^{i}\right)
$$

The solution of the differential equation is represented by $\mathrm{x}$, and the solution formula is as follows

$$
x=\sum_{i=0}^{\infty} x^{i}=I_{t_{0}}^{q} L \sum_{i=0}^{\infty} x^{i}+I_{t_{0}}^{q} \sum_{i=0}^{\infty} A^{i}+I_{t_{0}}^{q} \mathrm{~g}+x\left(t_{0}\right)
$$

Among them, $x_{\mathrm{i}}$ can be obtained by the following formula for further solving

$$
\left\{\begin{array}{l}
x^{0}=I_{t_{0}}^{q} g+x\left(t_{0}\right) \\
x^{1}=I_{t_{0}}^{q} L x^{0}+I_{t_{0}}^{q} A^{0}\left(x^{0}\right) \\
x^{2}=I_{t_{0}}^{q} L x^{1}+I_{t_{0}}^{q} A^{1}\left(x^{0}, x^{1}\right) \\
\cdots \\
x^{i}=I_{t_{0}}^{q} L x^{i-1}+I_{t_{0}}^{q} A^{i-1}\left(x^{0}, x^{1}, x^{2}, \cdots, x^{i-1}\right) \\
\cdots
\end{array}\right.
$$

\subsection{Fractional-order chaotic system and its solution}

A chaotic flow with a plane of equilibria system (CFPES) is proposed in Ref[41].The four-dimensional chaotic system equation is 


$$
\left\{\begin{array}{l}
\dot{x}_{1}=x_{2} \\
\dot{x}_{2}=x_{3} \\
\dot{x}_{3}=x_{3}+a y w-x_{3} x_{4} \\
\dot{x}_{4}=x_{1} x_{2}+b x_{2} x_{3}
\end{array}\right.
$$

The parameters of the system are $a$ and $b$. Based on conformable derivative, FCFPES can express as

$$
\left\{\begin{array}{l}
T_{t_{0}}^{q} x_{1}(t)=x_{2} \\
T_{t_{0}}^{q} x_{2}(t)=x_{3} \\
T_{t_{0}}^{q} x_{3}(t)=x_{3}+a y w-x_{3} x_{4} \\
T_{t_{0}}^{q} x_{4}(t)=x_{1} x_{2}+b x_{2} x_{3}
\end{array}\right.
$$

where $q$ represents the order of the system.

Decompose FCFPES according to formula (3) as follows

$$
\left[\begin{array}{l}
L_{x_{1}} \\
L_{x_{2}} \\
L_{x_{3}} \\
L_{x_{4}}
\end{array}\right]=\left[\begin{array}{l}
x_{2} \\
x_{3} \\
x_{3} \\
0
\end{array}\right],\left[\begin{array}{l}
N_{x_{1}} \\
N_{x_{2}} \\
N_{x_{3}} \\
N_{x_{4}}
\end{array}\right]=\left[\begin{array}{c}
0 \\
0 \\
a x_{2} x_{4}-x_{3} x_{4} \\
x_{1} x_{2}+b x_{2} x_{3}
\end{array}\right],\left[\begin{array}{l}
g_{1} \\
g_{2} \\
g_{3} \\
g_{4}
\end{array}\right]=\left[\begin{array}{l}
0 \\
0 \\
0 \\
0
\end{array}\right]
$$

Among them, the nonlinearity of the system is formed by the multiplication of two state variables. FCFPES contains similar nonlinear terms. According to Eq (5), one of the nonlinear terms $x_{1} x_{2}$ is decomposed as follows

$$
\left\{\begin{array}{l}
A_{1}^{0}=x_{1}^{0} x_{2}^{0} \\
A_{1}^{1}=x_{1}^{1} x_{2}^{0}+x_{1}^{0} x_{2}^{1} \\
A_{1}^{2}=x_{1}^{2} x_{2}^{0}+x_{1}^{1} x_{2}^{1}+x_{1}^{0} x_{2}^{2} \\
A_{1}^{3}=x_{1}^{3} x_{2}^{0}+x_{1}^{2} x_{2}^{1}+x_{1}^{1} x_{2}^{2}+x_{1}^{0} x_{2}^{3} \\
A_{1}^{4}=x_{1}^{4} x_{2}^{0}+x_{1}^{3} x_{2}^{1}+x_{1}^{2} x_{2}^{2}+x_{1}^{1} x_{2}^{3}+x_{1}^{0} x_{2}^{4}
\end{array}\right.
$$

here, letting the initial value $x_{0}=\left[x_{1}\left(t_{0}^{+}\right), x_{2}\left(t_{0}^{+}\right), x_{3}\left(t_{0}^{+}\right), x_{4}\left(t_{0}^{+}\right)\right]$. Then, the initial value can be described as

$$
\left\{\begin{array}{l}
x_{1}^{0}=x_{1}\left(t_{0}^{+}\right) \\
x_{2}^{0}=x_{2}\left(t_{0}^{+}\right) \\
x_{3}^{0}=x_{3}\left(t_{0}^{+}\right) \\
x_{4}^{0}=x_{4}\left(t_{0}^{+}\right)
\end{array} .\right.
$$


According to the two properties of the integrated integral operator, the iterative relation of the system is

$$
\begin{gathered}
\left\{\begin{array}{l}
c_{1}^{1}=c_{2}^{0} \\
c_{2}^{1}=c_{3}^{0} \\
c_{3}^{4}=c_{3}^{0}+a c_{2}^{0} c_{4}^{0}-c_{3}^{0} c_{4}^{0} \\
c_{4}^{1}=c_{1}^{0} c_{2}^{0}+b c_{2}^{0} c_{3}^{0}
\end{array} \quad \begin{array}{l}
c_{1}^{2}=c_{2}^{1} \\
c_{2}^{2}=c_{3}^{1} \\
c_{3}^{2}=c_{3}^{1}+a\left(c_{2}^{1} c_{4}^{0}+c_{2}^{0} c_{4}^{1}\right)-\left(c_{3}^{1} c_{4}^{0}+c_{3}^{0} c_{4}^{1}\right) \\
c_{4}^{2}=c_{1}^{1} c_{2}^{0}+c_{1}^{0} c_{2}^{1}+b\left(c_{2}^{1} c_{3}^{0}+c_{2}^{0} c_{3}^{1}\right)
\end{array}\right. \\
\left\{\begin{array}{l}
c_{1}^{3}=c_{2}^{2} \\
c_{2}^{3}=c_{3}^{2} \\
c_{3}^{3}=c_{3}^{2}+a\left(c_{2}^{2} c_{4}^{0}+2 c_{2}^{1} c_{4}^{1}+c_{2}^{0} c_{4}^{2}\right)-\left(c_{3}^{2} c_{4}^{0}+2 c_{3}^{1} c_{4}^{1}+c_{3}^{0} c_{4}^{2}\right) \\
c_{4}^{3}=c_{1}^{2} c_{2}^{0}+2 c_{1}^{1} c_{2}^{1}+c_{1}^{0} c_{2}^{2}+b\left(c_{2}^{2} c_{3}^{0}+2 c_{2}^{1} c_{3}^{1}+c_{2}^{0} c_{3}^{2}\right)
\end{array}\right. \\
\left\{\begin{array}{l}
c_{1}^{4} 14=c_{2}^{3} 23 ; \\
c_{2}^{4} 24=c_{3}^{3} 33 ; \\
c_{3}^{4} 34= \\
c_{4}^{4} 44=c_{3}^{3} 33+a\left(c_{2}^{3} c_{4}^{0}+3 c_{2}^{2} c_{4}^{1}+3 c_{2}^{1} c_{4}^{2}+c_{2}^{0} c_{4}^{3}\right)-\left(c_{3}^{3} c_{4}^{0}+3 c_{3}^{2} c_{4}^{1}+3 c_{3}^{1} c_{4}^{2}+c_{3}^{0} c_{4}^{3}\right) ;
\end{array}\right. \\
\left\{\begin{array}{l}
c_{1}^{5}=c_{2}^{4} c_{2}^{2}+c_{1}^{0} c_{2}^{3}+b\left(c_{2}^{3} c_{3}^{0}+3 c_{2}^{2} c_{3}^{1}+3 c_{2}^{1} c_{3}^{2}+c_{2}^{0} c_{3}^{3}\right) ; \\
\left.c_{2}^{5}=c_{3}^{4}\right) \\
c_{3}^{5}=c_{3}^{4}+a\left(c_{2}^{4} c_{4}^{0}+4 c_{2}^{3} c_{4}^{1}+6 c_{2}^{2} c_{4}^{2}+4 c_{2}^{1} c_{4}^{3}+c_{2}^{0} c_{4}^{4}\right)-\left(c_{3}^{4} c_{4}^{0}+4 c_{3}^{3} c_{4}^{1}+6 c_{3}^{2} c_{4}^{2}+4 c_{3}^{1} c_{4}^{3}+c_{3}^{0} c_{4}^{4}\right) ; \\
c_{4}^{5}=c_{1}^{4} c_{2}^{0}+4 c_{1}^{3} c_{2}^{1}+6 c_{1}^{2} c_{2}^{2}+4 c_{1}^{1} c_{2}^{3}+c_{1}^{0} c_{2}^{4}+b\left(c_{2}^{4} c_{3}^{0}+4 c_{2}^{3} c_{3}^{1}+6 c_{2}^{2} c_{3}^{2}+4 c_{2}^{1} c_{3}^{3}+c_{2}^{0} c_{3}^{4}\right) ;
\end{array}\right.
\end{gathered}
$$

(17)

So, the numerical solution of FCFPES obtained by CADM algorithm is

$$
x_{j}(t)=\sum_{i=0}^{5} c_{j}^{i} \frac{\left(t-t_{0}\right)^{i q}}{i ! q^{i}}
$$

here $j=1,2,3,4$. Ensure that the calculation accuracy after iteration is high enough, and the numerical solution can be calculated after five iterations. Setting $a=-1.1, b=0.92, q=0.9$, $h=0.01$ and initial value $x_{0}=[2.5,0,-1,-0.4]$. The Lyapunov exponents are $\mathrm{L}_{1}=0.2567, \mathrm{~L}_{2}=0$, $\mathrm{L}_{3}=0$, and $\mathrm{L}_{4}=-1.528$, and the Lyapunov dimension is $D_{\mathrm{L}}=3.1680 . \mathrm{L}_{1}$ is a positive Lyapunov exponent which means that this system is chaotic in this set of parameters. The phase diagrams on different planes of the system are shown in Fig. 1. 


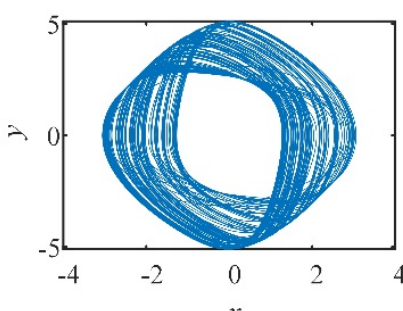

(a)

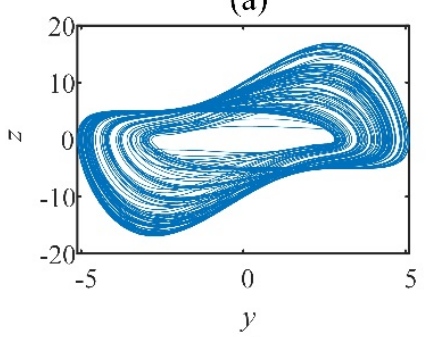

(d)

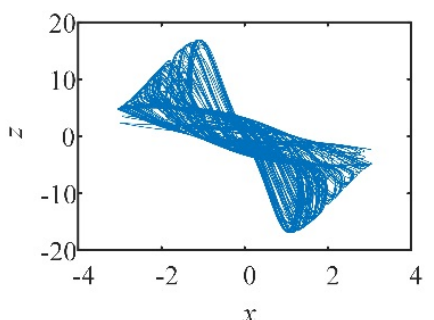

(b)

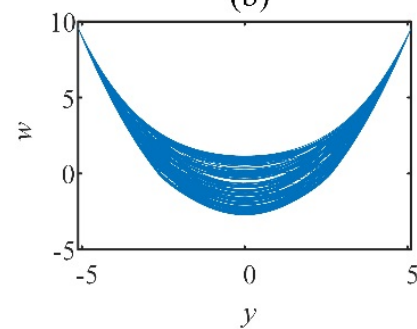

(e)

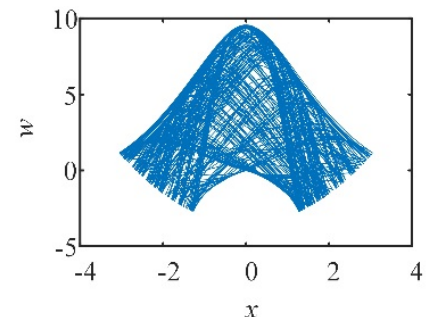

(c)

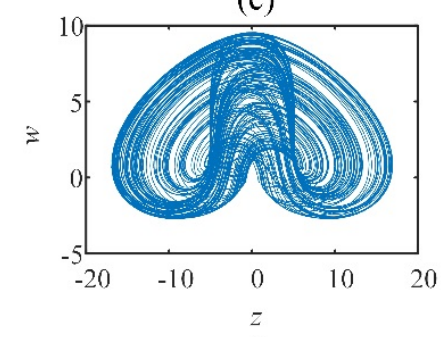

(f)

Fig. 1 Phase portraits of FCFPES ( $q=0.9$ ) (a) $x-y$ plane (b) $x-z$ plane (c) $x-w$ plane (d) $y-z$ plane(e) $y-w$ plane (f) $z-w$ plane

\section{Dynamical of different parameters of FCFPES}

\subsection{Stability analysis of fractional-order chaotic system}

Analyzing the stability at the equilibrium point is an indispensable research content of chaos theory in fractional chaotic systems. As we all know, the stability of the fractional order judgment balance point is inconsistent with the integer order. The necessary conditions for the asymptotic stability of the fractional order system using eigenvalues are given in Ref[42].

Lemma 1. The characteristic value satisfies the following conditions

$$
|\arg (\lambda)| \geq \frac{\pi \alpha}{2}, \alpha=\max \left(q_{i}, i=1,2,3\right)
$$

where $q$ is the order of the fractional order, and $\lambda$ is the eigenvalue. The eigenvalues of the system satisfy Theorem 1, which means the equilibrium point is stable. The stable area of the fractional chaotic system exceeds the imaginary axis and presents a fan-shaped area with an origin, as shown in Fig. 2. Obviously, if the eigenvalue is outside the fan-shaped area, the equilibrium point is unstable. The unstable equilibrium point is an important condition for the 
chaotic phenomenon of the system.

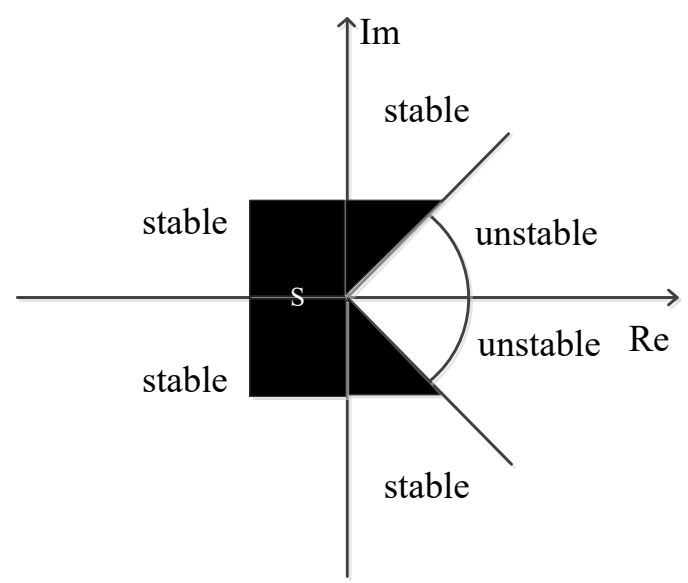

Fig. 2 Stability region of fractional-order system

Setting the left side of Eq. (1) to zero, FCFPES has a flat set of equilibrium points expressed as $E=\{(x, 0,0, w) \mid x, w \in R\}$. The Jacobian matrix $J$ of FCFPES can be described as

$$
J_{E}=\left[\begin{array}{cccc}
0 & 1 & 0 & 0 \\
0 & 0 & 1 & 0 \\
0 & a w & 1-w & 0 \\
0 & x & 0 & 0
\end{array}\right]
$$

The corresponding characteristic equation is

$$
\lambda^{2}\left(\lambda^{2}+(w-1) \lambda-a w\right)=0
$$

FCFPE always has the value of $\lambda_{1,2}$ as zero, and $\lambda_{3,4}$ are related to the initial value of $w$ and the system parameter $a$. Some cases and the corresponding characteristic values and their stability are list in Table1.

Table 1 Different values, balance point, eigenvalue and stability $(q=0.9)$

\begin{tabular}{ccccc}
\hline$a$ & $q$ & $w$ & $\lambda$ & stability \\
\hline & & 1 & $(0,0, i,-i)$ & Hopf branching point \\
& & -0.4 & $(1.64,0,0,-0.24)$ & Unstable focus \\
-1 & 0.9 & 1.1 & $(0,0,-0.05+1.0476 i,-0.05-1.0476 i)$ & Stable saddle-focus \\
& & 0.25 & $(0.38+0.3356 \mathrm{i}, 0.38+0.3356 \mathrm{i}, 0,0)$ & Unstable saddle-focus \\
\hline
\end{tabular}

\subsection{The analysis of dynamic characteristics evolves with parameters $a$ and $b$}

In this section, $a$ and $b$ are set as bifurcation parameters, and the evolution process of a 
chaotic system with order $q=0.9$ is analyzed through the bifurcation model and LEs. The fixed parameter $b=0.92$, and the LEs for $a \in(-1.7,-0.942)$ are shown in Fig. 3(a). Fig. 3(b) shows the bifurcation model, which corresponds well to the LES. Among them, FCFPES has a positive cycle doubling bifurcation at $a=-1.411$. After several period-doubling bifurcations, it enters the chaotic state at $a=-1.382$, and then enters the periodic window through some boundary crisis points and then enters the chaotic state again through the slicing and bifurcation. Finally, at $a=-0.991$, it branches from the reverse period doubling into the periodic state. The bifurcation behavior of parameter a is a typical chaotic bubble. Figs. 3(c) and 3(d) are the LEs and bifurcation models that vary with the parameter $b$. For $a=-1.1$, the positive period doubling bifurcation, edge crisis and split bifurcation can also be seen in Fig. 3(d). Table 2 gives detailed status to parameters $a$ and $b$.

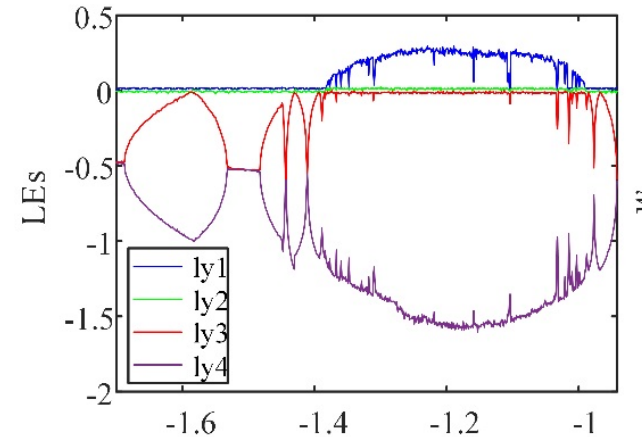

(a)

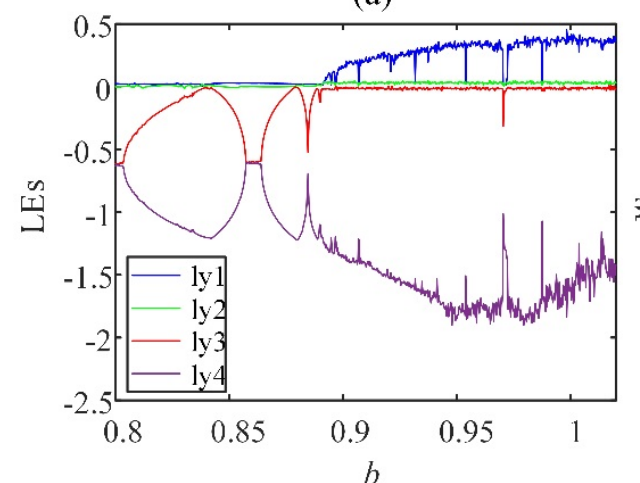

(c)

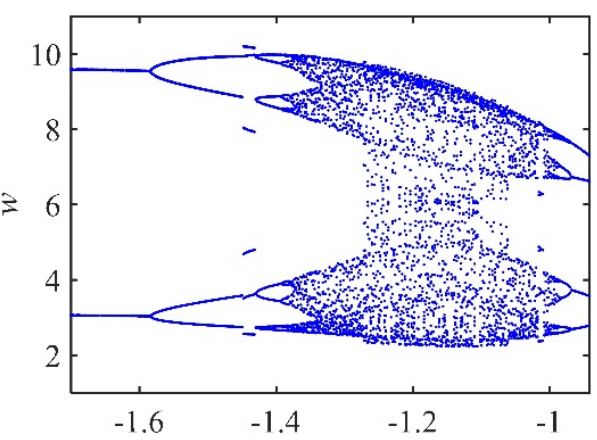

(b)

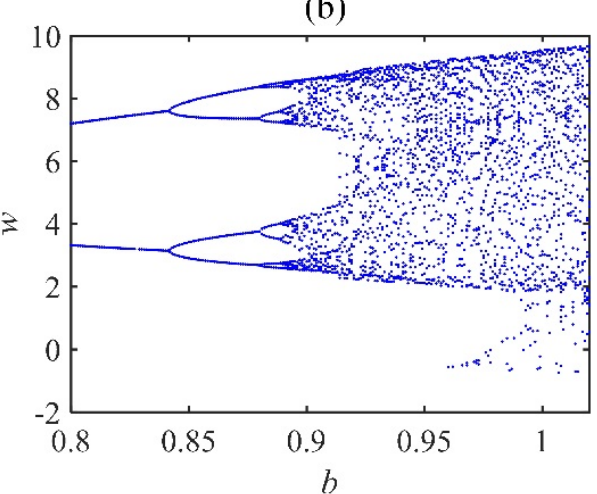

(d)

Fig. 3 Lyapunov exponent spectrum (LEs) and bifurcation diagram for different parameters. (a)-(b) The dynamics that varies with $a$ when $b=0.92$; (c)-(d) The dynamics that varies with $b$ when $a=-1.1$. 


\subsection{The analysis of dynamic characteristics evolves with order $q$}

FCFPES has a specific parameter $q$, which is used as a bifurcation parameter to study the evolution behavior of chaotic systems. The definition of conformable derivative satisfies the definition of integer order when the order $q$ is 1 . Therefore, this section can compare the integer-order and the fractional-order. Letting $a=-1.1$ and $b=0.92$, and the initial value is also $x_{0}=(2.5,0,-1,-0.4)$. In Fig. 4, the bifurcation diagram and Les are obtained. Obviously, FCFPES are all chaotic in $q \in(0.7,1)$. In addition, when $q$ gradually decreases, the largest Lyapunov exponent gradually increases from 0.26 to 1.15 , and the number of bifurcation points becomes denser. Obviously, FCFPES has a larger positive Ly value, which proves that the reconstructed FCFPES has a greater ratio of complexity and dynamic characteristics.

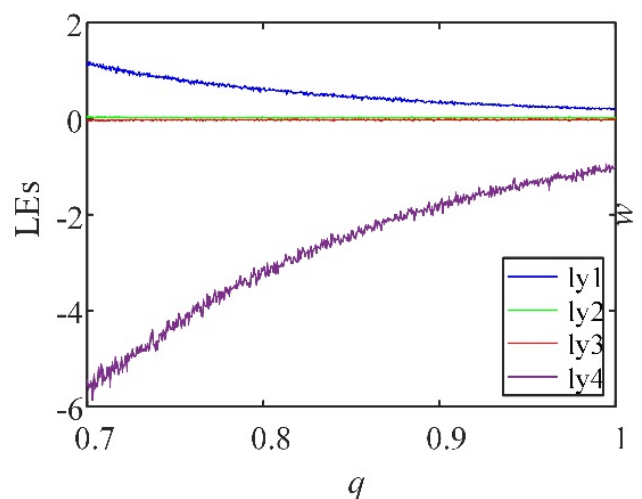

(a)

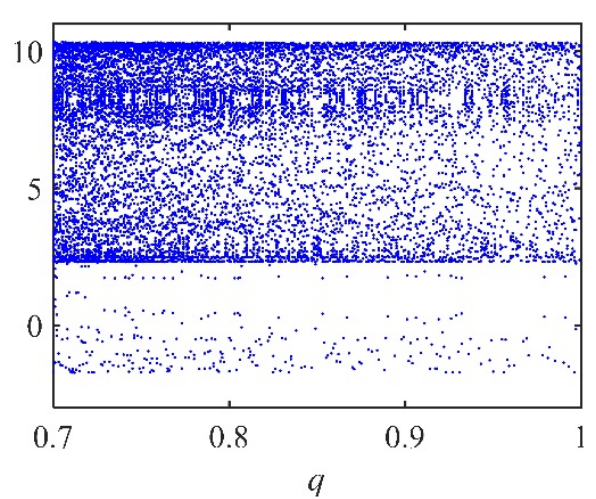

(b)

Fig. 4 Dynamical behaviors when $a=-1.1, b=0.92, q \in(0.7,1)$. (a) LEs; (b) bifurcation diagram

\subsection{Multi-stability analysis}

Multi-stability phenomena exist in some chaotic systems, and this special phenomenon plays a vital role in the field of information security. It means that the system has multiple sets of solutions when the parameter values remain unchanged. The bifurcation graph that vary with the initial value $x_{0}$ verifies the multi-stability in FCFPES. The fixed parameters $a=-1.1$, $b=0.92$ and $q=0.9$. Figure 5 is a bifurcation model, where (a), (b) and (c) correspond to $x_{1}=\left(k_{1}\right.$, $0,-1,-0.4), x_{2}=\left(2.5, k_{2},-1,-0.4\right)$ and $x_{3}=\left(2.5,0,-1, k_{3}\right)$. In Fig. 5(a), an obvious positive period-doubling bifurcation is observed, and the system state enters chaos from period through period-doubling bifurcation. In Fig. 5(b), there are periodic states and chaotic states, and they 
are symmetrical and discontinuous bifurcation models. Fig. 5(c) Chaos enters the periodic state through reverse period-doubling bifurcation behavior. With $k$ as the bifurcation parameter, each state is a different solution of the system, that is, the phenomenon of coexistence attractor. The coexistence phase diagram can explain this phenomenon more intuitively. The coexistence phenomena of different types of attractors including period-period, period-chaos and chaos-chaos coexistence phenomena are shown in Fig. 6. In particular, Fig. 6(b) is a heterogeneous chaotic coexistence attractor, and Fig. 6(d) is a heterogeneous periodic coexistence attractor. The above research shows that FCFPES has a relatively rich coexistence phenomenon, which will be more conducive to its application in confidential communication and other fields.
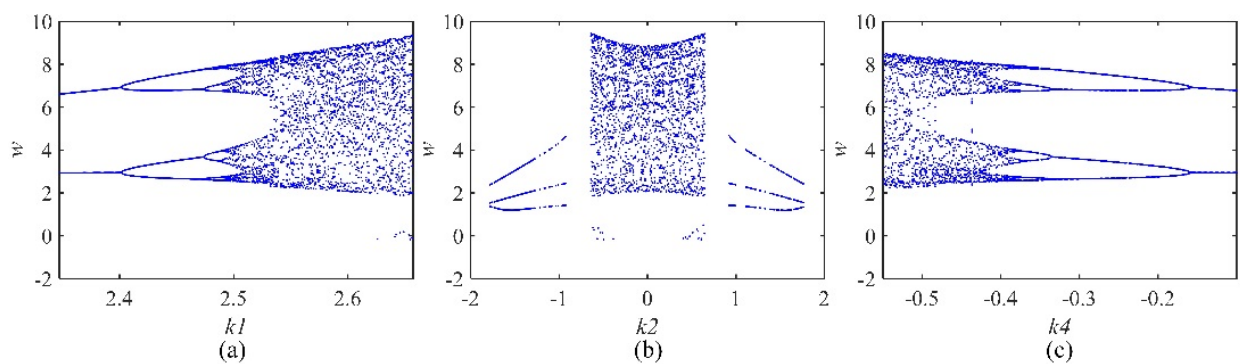

Fig. 5 Bifurcation model of $w$ that changes with the initial value. (a) $x_{1}=\left(k_{1}, 0,-1,-0.4\right)$; (b) $x_{2}=\left(2.5, k_{2},-1,-0.4\right) ;$ (c) $x_{3}=\left(2.5,0,-1, k_{3}\right)$.

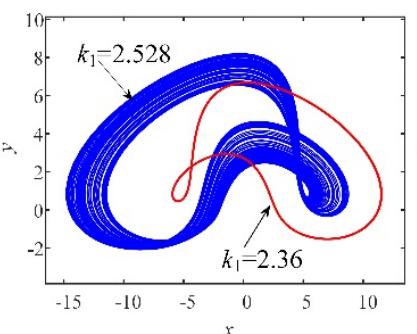

(a)

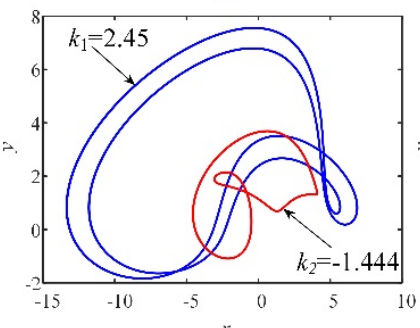

(d)

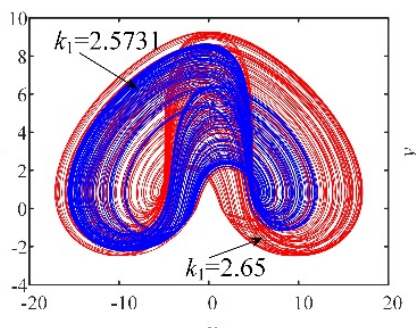

(b)

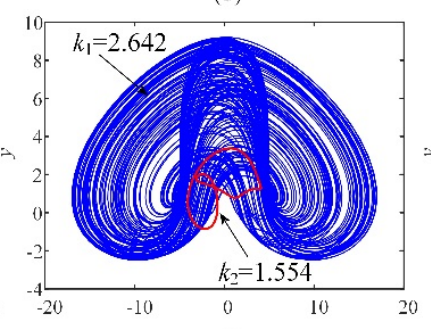

(c)

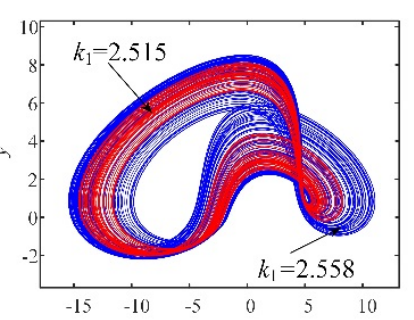

$\stackrel{x}{(c)}$

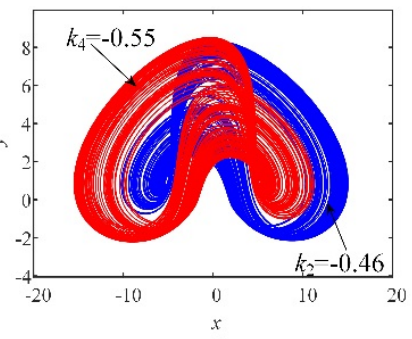

(f)

Fig. 6 Coexistence attractor on $x-y$ plane with different $k$. (a) $k_{1}=2.528, k_{1}=2.36$; (b) $k_{1}=2.5731$, 
$k_{1}=2.65$; (c) $k_{1}=2.515, k_{1}=2.558$; (d) $k_{1}=2.45, k_{2}=-1.444$; (e) $k_{1}=2.642, k_{2}=1.554$; (f) $k_{2}=-0.46$, $k_{4}=-0.55$.

\subsection{Attractor with different parameter plane}

The basin of attraction drawn based on the Lyapunov exponent can effectively reflect the state of the system. The system state distribution can be obtained from the attractor basin, and at the same time it also provides a selection instruction for its application. It should be noted that the parameter selection should avoid taking values at the boundary as much as possible, because the different calculation accuracy may cause the degradation and divergence of the system state. If it is used in image encryption and other fields, the value should be set within the orange range as much as possible for better encryption effect. Fig. 8 shows the attraction basins of two planes $a-q$ and $b-q$. The different states of the system are marked with different colors. In order to distinguish periodic, chaotic and divergent are represented by yellow, orange and blue respectively.

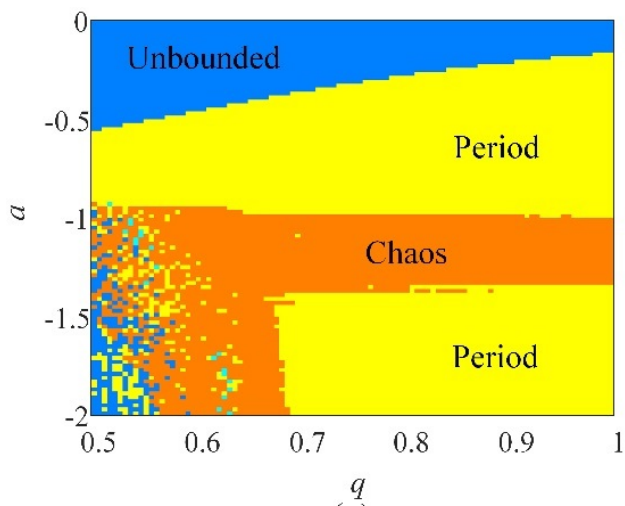

(a)

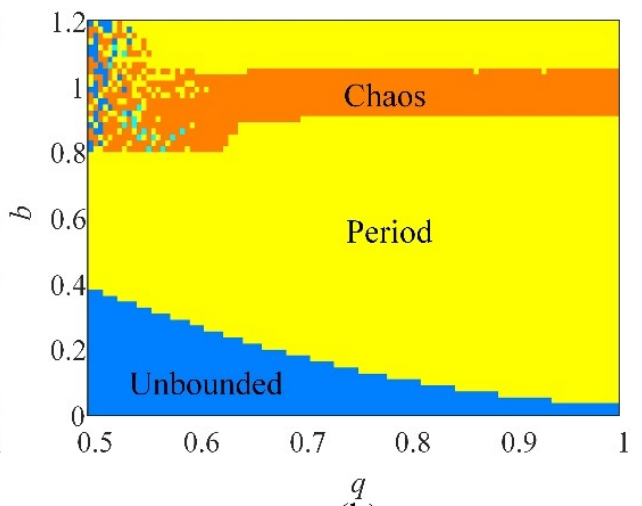

(b)

Fig. 7 Two-dimensional attractor basin (a) $b=0.92, a \in(-2,0), q \in(0.5,1)$; (b) $a=-1.1 ; b \in(0,1.2)$, $q \in(0.5,1)$.

\subsection{Complexity test of chaotic sequence}

The sequence generated by the chaotic system has special pseudo-randomness, which is an important performance of the application of the chaotic system. Therefore, it is necessary to measure the randomness of the sequence. The spectral entropy (SE) algorithm is improved from the Shannon entropy algorithm. The algorithm not only has a fast calculation speed and 
can better reflect the related structure of the sequence, but also can measure the complexity of the system more effectively. Especially when calculating continuous and stable time series, the advantage of the SE algorithm is more obvious. It should be pointed out that when calculating SE complexity, the first 20,000 data needs to be discarded to eliminate the influence of errors caused by system startup.

Fig. 8 is a 3D SE complexity diagram of different parameter planes with $x_{0}=(2.5,0,-1$, -0.4). The complexity graph on the $a-b$ plane with $q=0.9$ as shown in Fig. 8(a). In this region, the spectral entropy almost reaches 0.7 , which means that the higher the SE value, the better the pseudo-randomness of the sequence. Therefore, the selection parameter should try to take the value in the red area to ensure high complexity. Figs. 8(b) and 8(c) are the complexity diagrams on the $a-q$ plane and the $b-q$ plane. A similar trend is found from the two figures, that is, the complexity will increase correspondingly as $q$ decreases within a certain range. In addition, when $a$ or $b$ is unchanged, SE can take the lowest value at $q=1$. In other words, FCFPES have higher complexity and better random performance than integer-order chaotic systems. Meanwhile, the complexity graph provides some basis for the selection of parameters.

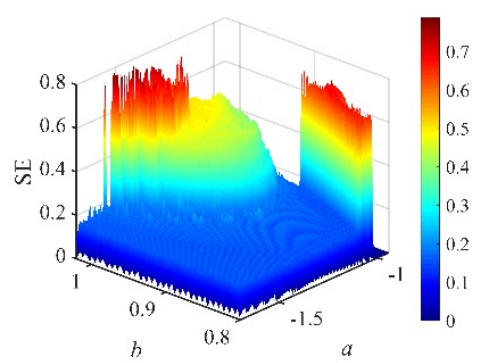

(a)

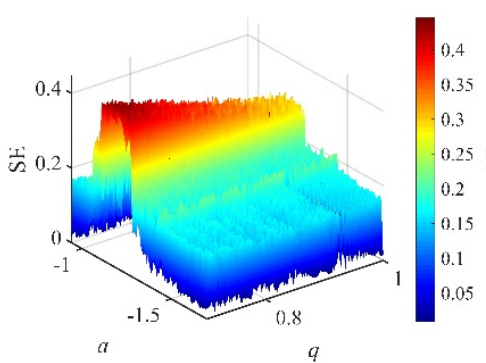

(b)

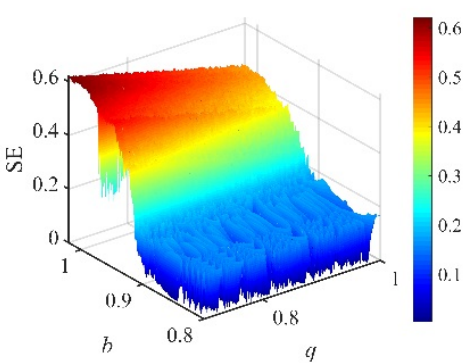

(c)

Fig. 8 Spectral Entropy (a)on $a-b$ plane; (b)on $a-q$ plane; (c)on $b-q$ plane.

\section{Chaos-based PRNG}

Chaos theory has applications in many fields, among which it can be designed to be a pseudo-random number generator (PRNG) with pseudo-randomness in its generated sequence. The requirements of PRNGs appear in many encryption applications. The chaotic system with multiple stability can be used to design a PRNG with good performance. In the previous section, we observed the high sensitivity of CFPES by changing its initial value by generating various coexistence attractors. Therefore, three sets of parameters are selected in the higher 
numerical area in the complexity graph to construct the PRNG respectively. Here, a new PRNG generation algorithm is proposed based on FCFPES. Considering the time consumed by iterative calculations and the amount of data that needs to be generated to achieve fast calculations on hardware, our experiment is based on MATLAB to generate 16-bit significant digital PRNG process as shown in Fig9.

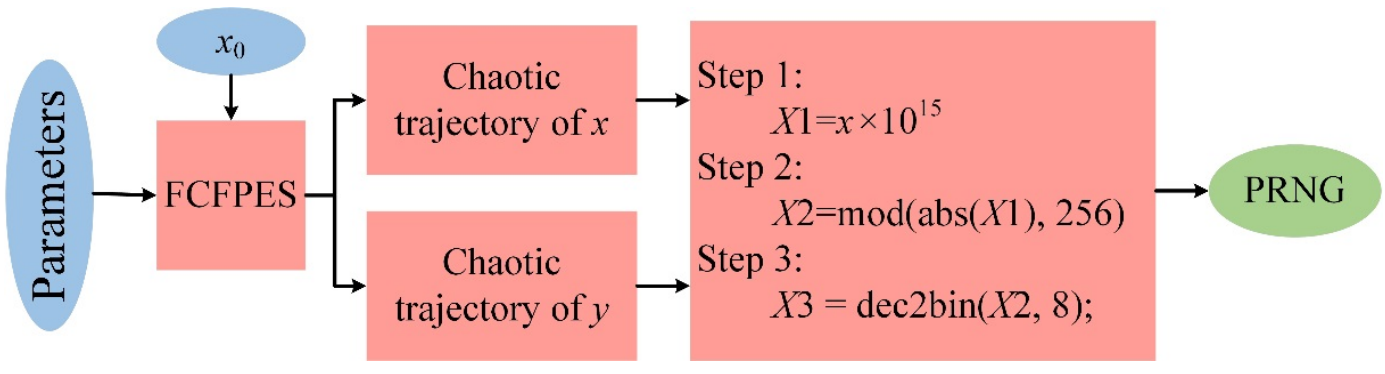

Fig. 9 The flowchart of generating PRNG

Our experiment uses statistical software NIST-800-22, which is used for statistical testing to test the randomness of PRNG. NIST-800-22 contains multiple empirical statistical tests, which can provide a true assessment of the randomness of PRNG. Generally speaking, when $p>0.01$ obtained by testing a binary sequence, we think PRNG has passed the NIST test. According to the algorithm, when the parameters are regarded as input, we can obtain three PRNGs, corresponding to the parameters $(a, b, q)=(-1.189,0.512,0.9),(-1.134,0.92,0.8)$ and $(-1.1$, $1.019,0.7)$. The data used for measurement is divided into 100 groups, each of which is a 1,000,000-bit binary sequence. The $p$-values of PRNG1, PRNG2 and PRNG3 tested by NIST-800-22 are listed in Table 3. Obviously, all $p$-values are greater than 0.01 , that is, three PRNGs pass all statistical tests.

Table 3. NIST tests results of FCFPES.

\begin{tabular}{c|c|ccc|c|}
\hline \multicolumn{5}{|c|}{ Each group contains 1,000,000 dates } \\
\hline & NIST tests & PRNG1 & PRNG2 & PRNG3 & \multirow{2}{*}{ Result } \\
\cline { 2 - 5 } & & 0.3041 & 0.0210 & 0.0909 & Random \\
\hline 1. & Block frequency & 0.6787 & 0.7198 & 0.3505 & Random \\
2. & Frequency & 0.0909 & 0.4373 & 0.7598 & Random \\
3. & FFT & 0.3505 & 0.1154 & 0.2023 & Random \\
4. & Approximate entropy & 0.9569 & 0.0762 & 0.8248 & Random \\
5. & Cumulative sums & 0.8311 & 0.4197 & 0.4343 & Random \\
6. & Serial-1 & 0.7981 & 0.1626 & 0.6163 & Random \\
7. & Runs & 0.7792 & 0.3041 & 0.4190 & Random \\
8. & Longest run of ones & & & &
\end{tabular}




\begin{tabular}{c|c|llll}
9. & Overlapping template & 0.4012 & 0.4373 & 0.8343 & Random \\
10. & Non-overlapping template & 0.4930 & 0.4996 & 0.5164 & Random \\
11. & Linear complexity & 0.9717 & 0.6993 & 0.7197 & Random \\
12. & Binary matrix rank & 0.8514 & 0.7792 & 0.0519 & Random \\
13. & Universal statistical & 0.3838 & 0.9717 & 0.9114 & Random \\
14. & Random excursions & 0.4621 & 0.3578 & 0.3232 & Random \\
15. & Random Excursions Variant & 0.4217 & 0.3534 & 0.3808 & Random \\
\hline
\end{tabular}

\section{DSP implementation}

Compared with analog circuits, digital circuits have faster processing speed, high precision and reliability. Therefore, the realization of the digital circuit of the chaotic system is more reliable. In this section, we use DSP to implement CFPES. We choose the DSP chip model as TMS320F28335. The chip's operating speed reaches $150 \mathrm{MHz}$ and 16-bit floating point operations can also meet the required accuracy requirements. In addition, a DA converter is needed to convert the digital signal output by the DSP into an analog signal and import it into the oscilloscope. The model of the D/A converter is DAC8552. Fig. 10 shows a schematic diagram of each hardware connection. $\mathrm{C}$ language is used to design the software design of the chaotic system. Fig. 11 shows the 1DSP platform designed and implemented.

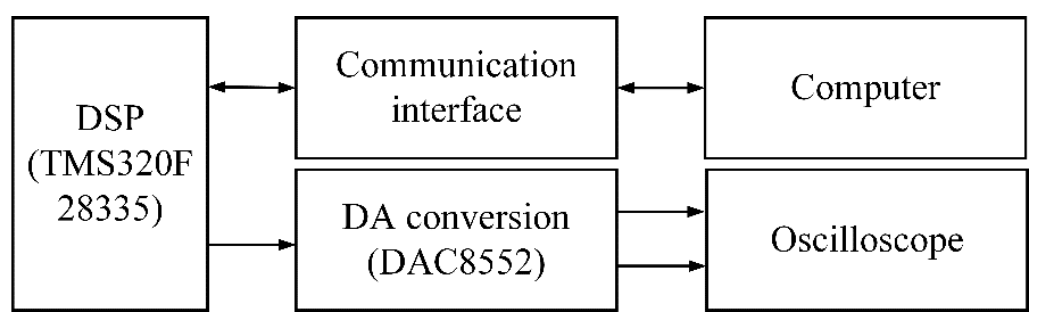

Fig. 10 DSP connection diagram.

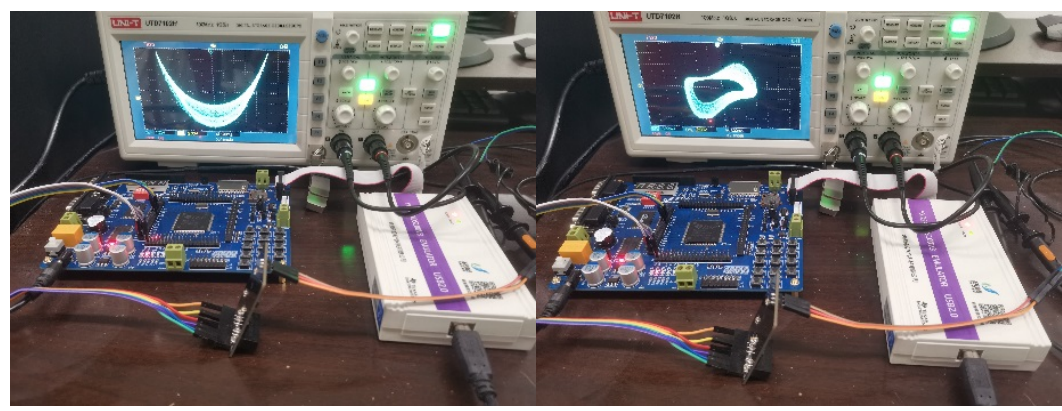


Fig. 11 Digital circuit experimental platform

The DSP implementation parameters are set as, $h=0.01, x_{0}=(2.5,0,-1,-0.4), a=-1.1, b=$ 0.92 , and $q=0.9$. It should be pointed out that this set of parameters is the same as the attractor parameters in Fig. 1. The oscilloscope shows the attractor of the signal after D/A conversion on a plane. Adjust the oscilloscope to take a screenshot, and the result is shown in Figure 12. The experimental results obtained the approximate chaotic attractors.

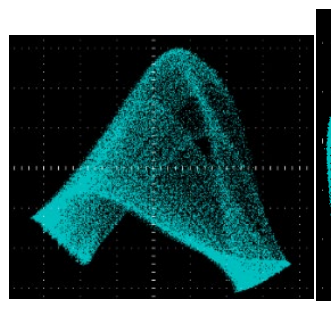

(a)

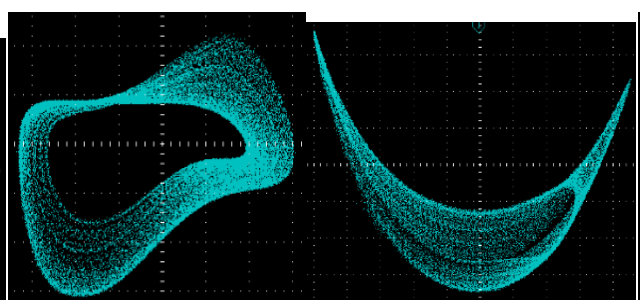

(b)

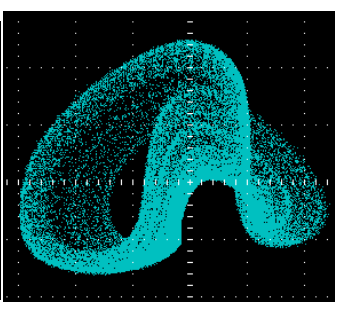

(c)

(d)

Fig. 12 Chaotic attractors on DSP platform

\section{Conclusions}

In this paper, a new four-dimensional fractional-order chaotic system is constructed and its numerical solution are obtained by CADM. The balance point set of the system is distributed on a plane. It is interesting that the stability at the equilibrium point is affected by the initial value. When the system changes with parameters, some typical bifurcations appear, such as positive and negative cycle doubling bifurcations, slicing bifurcations, and boundary crises, showing abundant dynamics. When changing with the order $q$, the value of LEs reaches the maximum value at $q=0.7$. The dynamic behavior changing with the parameters indicates that FCFPES has a higher complexity chaotic sequence and richer dynamic evolution behavior. In addition, the multi-stable phenomenon of FCFPES is studied through the coexistence phase diagram and bifurcation model, which includes the asymmetric coexistence periodic attractor and coexistence attractor, and the symmetric coexistence chaotic attractor phenomenon. The basin of attraction drawn based on LE provides a basis for the selection of the system parameters. The reconstructed fractional-order chaotic system still has multiple stability and different coexistence types. Then, the three PRNGs were tested using NIST-800-22, and the results showed that they all passed all statistical tests. Finally, the realization of the DSP platform demonstrates the feasibility of the system. This paper provides a certain research foundation for the research of fractional chaotic systems, and also provides new ideas for the 
application of fractional chaotic systems.

\section{Acknowledgements}

This work is supported by Scientific Research Funding Project of the Educational Department of Liaoning Province, China (J2020051).

\section{References}

[1] S. Mukhopadhyay and S. Banerjee, "Learning dynamical systems in noise using convolutional neural networks," Chaos, vol. 30, no. 10, p. 103125, Oct 2020, doi: 10.1063/5.0009326.

[2] L. Zhang, K. Sun, S. He, H. Wang, and Y. Xu, "Solution and dynamics of a fractional-order 5-D hyperchaotic system with four wings," The European Physical Journal Plus, vol. 132, no. 1, 2017, doi: 10.1140/epjp/i2017-11310-7.

[3] M. Wang, Y. Deng, X. Liao, Z. Li, M. Ma, and Y. Zeng, "Dynamics and circuit implementation of a four-wing memristive chaotic system with attractor rotation," International Journal of Non-Linear Mechanics, vol. 111, pp. 149-159, 2019, doi: 10.1016/j.jijnonlinmec.2019.02.009.

[4] K. Sun, X. I. A. Wang, and J. C. Sprott, "Bifurcations and Chaos in Fractional-Order Simplified Lorenz System," International Journal of Bifurcation and Chaos, vol. 20, no. 04, pp. 1209-1219, 2010, doi: 10.1142/s0218127410026411.

[5] Chenguang et al., "Coexistence of multiple attractors for an incommensurate fractional-order chaotic system," The European Physical Journal Plus, vol. 135, no. 1, pp. 1-21, 2020.

[6] C. Ma, J. Mou, Y. Cao, T. Liu, and J. Wang, "Multistability analysis of a conformable fractional-order chaotic system," Physica Scripta, vol. 95, no. 7, 2020.

[7] S. Eshaghi, "Synchronize and control chaos of chaotic fractional Laser system," in The Third Conference on Chaotic Dynamical Systems, 2020.

[8] M. Farman, A. Akgül, M. U. Saleem, S. Imtiaz, and A. Ahmad, "Dynamical behaviour of fractional-order finance system," Pramana, vol. 94, no. 1, pp. 1-10, 2020.

[9] S. He, K. Sun, and S. Banerjee, "Dynamical properties and complexity in fractional-order diffusionless Lorenz system," The European Physical Journal Plus, vol. 131, no. 8, 2016, doi: 10.1140/epjp/i2016-16254-8.

[10] C. Liu, X. Cai, Y. Wang, L. Liu, Y. Wang, and G. Zheng, "Fractional-Order Hidden Attractor Based on the Extended Liu System," Mathematical Problems in Engineering, vol. 
2020, 2020.

[11] A. Khan, L. S. Jahanzaib, and P. Trikha, "Changing Dynamics of the First, Second and Third Approximations of the Exponential Chaotic System and Their Application in Secure Communication Using Synchronization," International Journal of Applied \& Computational Mathematics, vol. 7, no. 1, 2021.

[12] N. A. A. Fataf, S. K. Palit, S. Mukherjee, M. R. M. Said, D. H. Son, and S. Banerjee, "Communication scheme using a hyperchaotic semiconductor laser model: Chaos shift key revisited," European Physical Journal Plus, vol. 132, no. 11, p. 492, 2017.

[13] F. Yang, J. Mou, K. Sun, and R. Chu, "Lossless image compression-encryption algorithm based on BP neural network and chaotic system," Multimedia Tools \& Applications, vol. 79, no. 1-2, 2020 .

[14] F. Yang, J. Mou, C. Luo, and Y. Cao, "An improved color image encryption scheme and cryptanalysis based on hyperchaotic sequence," Physica Scripta, 2019.

[15] A. N. Kengnou Telem, D. Tchiotsop, T. Kanaa, H. B. Fotsin, and D. Wolf, "A robust chaotic fast Walsh transform encryption for gray scale biomedical image transmission," Signal \& Image Processing An International Journal, vol. 6, no. 3, pp. 81-102, 2015.

[16] F. T. Arecchi, R. Meucci, P. GP, and T. JR, "Experimental Evidence of Subharmonic Bifurcations, Multistability, and Turbulence in a Q-Switched Gas Laser," Physical Review Letters, vol. 49, p. 1217, 1982.

[17] H. Natiq, M. R. M. Said, M. R. K. Ariffin, S. He, L. Rondoni, and S. Banerjee, "Self-excited and hidden attractors in a novel chaotic system with complicated multistability," The European Physical Journal Plus, vol. 133, no. 12, 2018, doi: 10.1140/epjp/i2018-12360-y.

[18] F. Yu et al., "Multistability Analysis, Coexisting Multiple Attractors, and FPGA Implementation of Yu-Wang Four-Wing Chaotic System," Mathematical Problems in Engineering, vol. 2020, p. 7530976, 2020/08/29 2020, doi: 10.1155/2020/7530976.

[19] J.-J. He and B.-C. Lai, "A novel 4D chaotic system with multistability: Dynamical analysis, circuit implementation, control design," Modern Physics Letters B, vol. 33, no. 21, p. 1950240, 2019, doi: 10.1142/s0217984919502403.

[20] F. Li, C. Tai, H. Bao, J. Luo, and B. Bao, "Hyperchaos, quasi-period and coexisting behaviors in second-order-memristor-based jerk circuit," The European Physical Journal Special Topics, vol. 229, no. 6-7, pp. 1045-1058, 2020, doi: 10.1140/epjst/e2020-900123-5.

[21] B. Bao et al., "Chaotic Bursting Dynamics and Coexisting Multistable Firing Patterns in 3D Autonomous Morris-Lecar Model and Microcontroller-Based Validations," International Journal of Bifurcation and Chaos, vol. 29, no. 10, 2019, doi: $10.1142 / \mathrm{s} 0218127419501347$. 
[22] C. Ma, J. Mou, F. Yang, and H. Yan, "A fractional-order hopfield neural network chaotic system and its circuit realization," The European Physical Journal Plus, vol. 135, no. 1, p. 100, 2020/01/23 2020, doi: 10.1140/epjp/s13360-019-00076-1.

[23] F. Yu et al., "Dynamic Analysis, Circuit Design, and Synchronization of a Novel 6D Memristive Four-Wing Hyperchaotic System with Multiple Coexisting Attractors," Complexity, vol. 2020, p. 5904607, 2020/05/19 2020, doi: 10.1155/2020/5904607.

[24] T. Liu, J. Yu, H. Yan, and J. Mou, "A Fractional-Order Chaotic System With Infinite Attractor Coexistence and its DSP Implementation," IEEE Access, vol. 8, pp. 199852-199863, 2020, doi: 10.1109/access.2020.3035368.

[25] K. Rajagopal, A. J. M. Khalaf, Z. Wei, V.-T. Pham, A. Alsaedi, and T. Hayat, "Hyperchaos and Coexisting Attractors in a Modified van der Pol-Duffing Oscillator," International Journal of Bifurcation and Chaos, vol. 29, no. 05, 2019, doi: $10.1142 / \mathrm{s} 0218127419500676$.

[26] C. Li, W. Joo-Chen Thio, J. C. Sprott, H. H.-C. Iu, and Y. Xu, "Constructing Infinitely Many Attractors in a Programmable Chaotic Circuit," IEEE Access, vol. 6, pp. 29003-29012, 2018, doi: 10.1109/access.2018.2824984.

[27] S. Qureshi, A. Atangana, and A. A. Shaikh, "Strange chaotic attractors under fractal-fractional operators using newly proposed numerical methods," The European Physical Journal Plus, vol. 134, no. 10, 2019, doi: 10.1140/epjp/i2019-13003-7.

[28] C. Li, F. Min, and C. Li, "Multiple coexisting attractors of the serial-parallel memristor-based chaotic system and its adaptive generalized synchronization," Nonlinear Dynamics, 2018.

[29] F. Yu et al., "A New 4D Four-Wing Memristive Hyperchaotic System: Dynamical Analysis, Electronic Circuit Design, Shape Synchronization and Secure Communication," International Journal of Bifurcation and Chaos, vol. 30, no. 10, p. 2050147, 2020.

[30] A. B. A, K. R. B, A. J. M. K. C, S. J. A, G. D. L. D. E, and J. K. D, "Dynamical analysis of a new multistable chaotic system with hidden attractor: Antimonotonicity, coexisting multiple attractors, and offset boosting," Physics Letters A, vol. 383, no. 13, pp. 1450-1456, 2019.

[31] D. Peng, K. H. Sun, and A. O. A. Alamodi, "Dynamics analysis of fractional-order permanent magnet synchronous motor and its DSP implementation," International Journal of Modern Physics B, vol. 33, no. 06, p. 1950031, 2019, doi: 10.1142/s0217979219500310.

[32] S. He, K. Sun, X. Mei, B. Yan, and S. Xu, "Numerical analysis of a fractional-order chaotic system based on conformable fractional-order derivative," The European Physical Journal Plus, vol. 132, no. 1, 2017, doi: 10.1140/epjp/i2017-11306-3. 
[33] R. Khalil, M. A. Horani, A. Yousef, and M. Sababheh, "A new definition of fractional derivative," Journal of Computational and Applied Mathematics, vol. 264, 2014.

[34] J. Ruan, K. Sun, J. Mou, S. He, and L. Zhang, "Fractional-order simplest memristor-based chaotic circuit with new derivative," European Physical Journal Plus, vol. 133, no. 1, p. 3, 2018.

[35] O. Acan and D. Baleanu, "A new numerical technique for solving fractional partial differential equations," Miskolc Mathematical Notes, vol. 19, no. 1, 2018, doi: 10.18514/mmn.2018.2291.

[36] S. Bhalekar, V. Daftardar-Gejji, D. Baleanu, and R. Magin, "Transient chaos in fractional Bloch equations," Computers \& Mathematics with Applications, vol. 64, no. 10, pp. 3367-3376, 2013

[37] Y. Li, Z. Li, M. Ma, and M. Wang, "Generation of grid multi-wing chaotic attractors and its application in video secure communication system," Multimedia Tools \& Applications, vol. 79, no. 1, 2020.

[38] H. Wang, K. Sun, and S. He, "Characteristic Analysis and DSP Realization of Fractional-Order Simplified Lorenz System Based on Adomian Decomposition Method," International Journal of Bifurcation and Chaos, vol. 25, no. 06, p. 1550085, 2015, doi: $10.1142 / \mathrm{s} 0218127415500856$.

[39] S. Dai, K. Sun, W. Ai, and Y. Peng, "Novel discrete chaotic system via fractal transformation and its DSP implementation," Modern Physics Letters B, p. 2050429, 2020.

[40] C. L. Li, C. Q. Liu, Y. G. He, and X. B. Yang, "Analysis, Stabilization, and DSP-Based Implementation of a Chaotic System with Nonhyperbolic Equilibrium," Complexity, vol. 2020, 2020.

[41] A. Bayani, K. Rajagopal, A. J. M. Khalaf, S. Jafari, G. D. Leutcho, and J. Kengne, "Dynamical analysis of a new multistable chaotic system with hidden attractor: Antimonotonicity, coexisting multiple attractors, and offset boosting," Physics Letters A, vol. 383, no. 13, pp. 1450-1456, 2019, doi: 10.1016/j.physleta.2019.02.005.

[42] E. et al., "Equilibrium points, stability and numerical solutions of fractional-order predator-prey and rabies models - ScienceDirect," Journal of Mathematical Analysis \& Applications, vol. 325, no. 1, pp. 542-553, 2007. 